\title{
IMU BASED SMARTPHONE CONTROLLED HUMAN FOLLOWING ROBOT WITH FALL DETECTION
}

\author{
Dr. Mahesh K \\ Professor, Dept of EEE \\ Sir MVIT, Bengaluru, Karnataka, INDIA
}

\author{
Sathya Naresh, VC Praveen \\ Student, Dept of EEE \\ Sir MVIT, Bengaluru, Karnataka, INDIA
}

\begin{abstract}
The advancement in robotic technology has improved drastically in the past decade, from load carrying to assembling of huge aircrafts has made it easier and has reduced human labour. Furthermore, with technologies like Artificial Intelligence and Machine Learning becoming pervasive robots are tending to grow smarter. Human following robots employing RFID and vision techniques like camera and laser are current trend in the market. But they all are mainly based on line of sight which implies that it cannot reacquire the target when lost. In this paper, a novel method is employed to obtain the human following feature using Inertial Measurement Unit (IMU). Unlike the former techniques, using IMU would not pose any shortcomings of losing the target. The most important feature needed is the ability for the robot to follow that particular person. The application called the Hyper IMU is utilised to convert a regular Smartphone into a powerful IMU device. The availability of the sensors which are already embedded in the Smartphone these days is highly advantageous for the implementation of human following robot. The robot tracks the movement of the human and heads in the same direction as guided by the sensors. The implementation of ultrasonic sensor is to obtain obstacle detection which can avoid damages to the robot in case of its absence.
\end{abstract}

Keywords - IMU, Ultrasonic sensor, HyperIMU, Smartphone, GSM, Fall Detection

\section{INTRODUCTION}

Every time the robot gets advanced, the usefulness of it increases tremendously. Krueger V., et al.(2016) have emphasized in their work that Robotic technology has advanced appreciably in the recent years. The reason being their increased efficiency and practical ability to work. However, these robots have only found their existence in industries and smart human friendly robots for general purpose use have yet to be achieved. To realize this, interactions between these robots and the environment should be more autonomous rather than programmed to do a couple of tasks. Pradeep B. V et al.(2017) have openly stated in their paper that communication is an important aspect for this purpose and also in the design and development of human friendly robots. Closer the both, easier is this interaction. As stated by Marina Md. Din et al.(2018) although there are outdoor localization techniques such as GPS and GLONASS, they are not the best solutions to carry out target following feature. This limitation occurs due to the fact that these signals have low penetration capability through walls of buildings and other structures. Since a large time of our day is spent indoors, a better alternative to cater this problem is much required. Hence, as concluded by Suresh kumar R. et al.(2018) in their work a viable indoor tracking technique which is also easily affordable is required. Since Smartphones are ubiquitous and there is no one person who lives without using it. Therefore, in this proposed project we have considered it to be the best resource that can be tapped to comfort the problem.

\section{Fall Detection}

Honig S. S. et al(2018) and Ren L., et al.(2019), the authors from both the papers have admitted that it is a major challenge in the public healthcare domain, especially for the elderly person as the decline of their physical fitness, and timely and reliable surveillance is necessary to mitigate the negative effects of falls. According to the study conducted by Hu J.-S., et al.(2014) the Human Following Robot can monitor the movements of human body and recognize a fall from normal daily activities. From the Smartphone application, the IMU coordinates can be read and the robot can be programmed to alert the emergency contact number which will be preinstalled Decker, M., et al.(2017) and Fukuda T et al.(2011)

The rest of the paper is organized as follows. Proposed embedding and extraction algorithms are explained in section II. Experimental results are presented in section III. Concluding remarks are given in section IV. 


\section{Block Diagram}

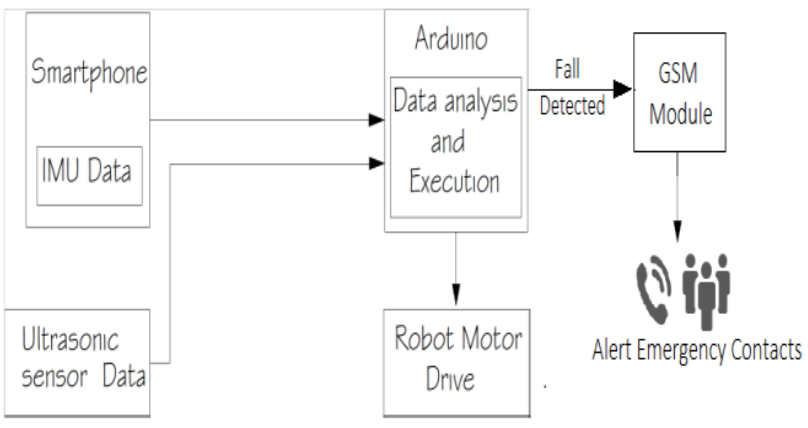

Fig. 1. Block Diagram

Components of the block diagram are as shown in Fig 1:

1. Smartphone pre-installed with HyperIMU application

2. Ultrasonic sensor

3. Arduino UNO

4. Robot motor drive

5. GSM Module

\section{COMPONENT DETAILS}

\section{A. HyperIMU Smartphone App}

The smartphone application as shown in Fig 2 would be used for this project and is called as HyperIMU which is easily available in google playstore. It is an application which turns the smartphone into a powerful Inertial Measurement Unit(IMU) device. Kianifar R et al.(2019) and Morioka K et al.(2004) have worked on distributed sensors such as IMU and believe that it could be accessed to obtain movement data. This application is used to collect sensory data from the smartphones. Accelerometer, Gyroscope and Magnetometer are the few sensors of the mobile that will be accessed by this app. It exposes this collected data via network protocols TCP, UDP. It can also collect them into a file for offline processing.

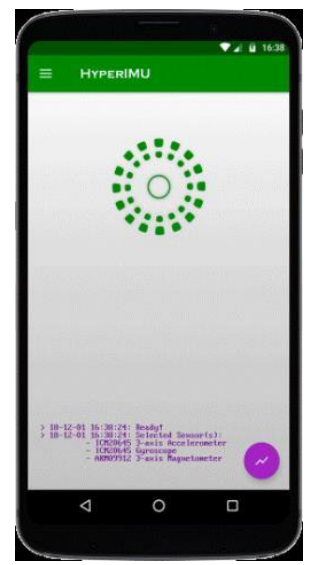

Fig. 2. HyperIMU Smartphone Application

\section{B. Gyroscope}

It consists of wheels or discs which can spin along its axis with respect to its position. These gyroscopes are especially used in aviation. This sensor is used in navigation systems and this can be helpful for the robot to navigate in the direction of the specified target by the change in pitch and roll.

\section{Accelerometer}

These sensors are used to measure acceleration of a moving body. The use of accelerometer in drones has helped in stabilization. The use of accelerometer in this project can help determine the robot the acceleration of the target in the given direction and moves according to the data.

\section{Magnetometer}

This sensor is used to measure magnetism or change in magnetic field at a location. The use of these sensors has increased in integrated circuits. The magnetometer measures the magnetism and helps the robot to move in the given direction as per the command received.

\section{E. Ultrasonic Sensor}

The ultrasonic sensor as shown in Fig 3 measures the point to point distances. It consists of transmitter, receiver and sometimes transceivers. The transmitter converts electrical signals to ultrasound. The receiver converts the ultrasound to electrical sensor. The use of ultrasonic is for obstacle detection which can otherwise result in damaging the robot from crashing onto a surface.

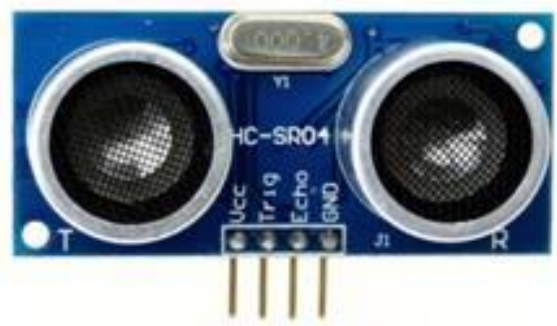

Fig. 3. Ultrasonic Sensor HC-SR04

\section{F. Arduino UNO}

Arduino Uno is the first series USB based Arduino board developed by Arduino.cc. It is programmed using software called the Arduino IDE. It can be powered using a external battery or by USB cable. Arduino UNO as shown in Fig 4 is used because programming the robot is lot simpler and matches our simple robot requirements. 


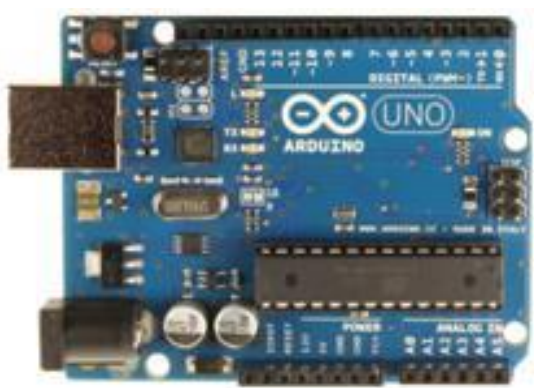

Fig. 4. Arduino UNO

\section{G. Motor Drive}

The motor driver as shown in Fig 5 helps in predetermining the manner in which the motor must run. It helps in increasing or decreasing the voltage or current depending on the specification of the given motor. It is used in places where the availability of the power is lower than the power required by the motor.

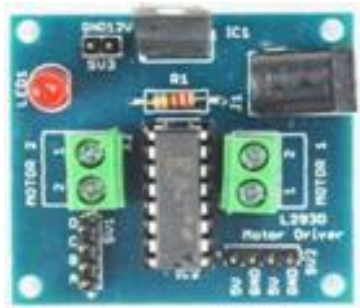

Fig. 5. Motor Drive L293D

\section{H. GSM Module}

GSM module as shown in Fig 6 is a chip used to establish communication between the robot and the human. The robot which guides the human detects the fall and immediately contacts the emergency contact number pre-installed in the robot.

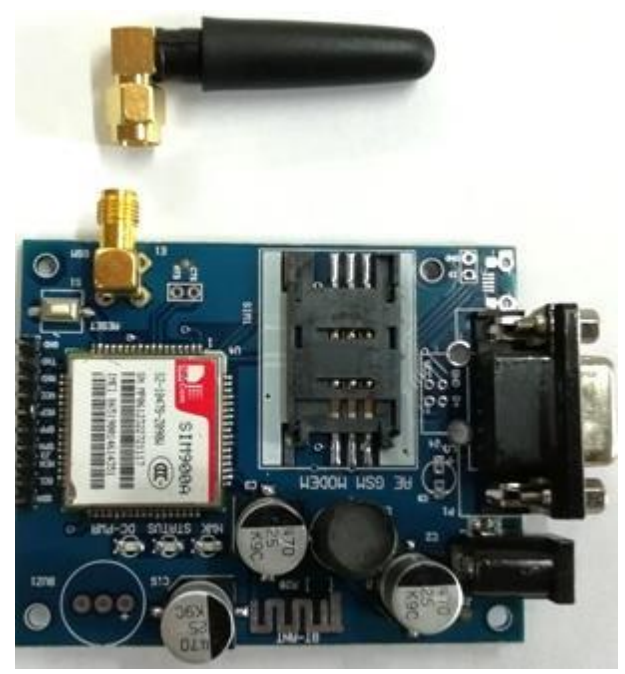

Fig. 6. GSM Module SIM900A

\section{METHODOLOGY}

\section{A. Flow Diagram}

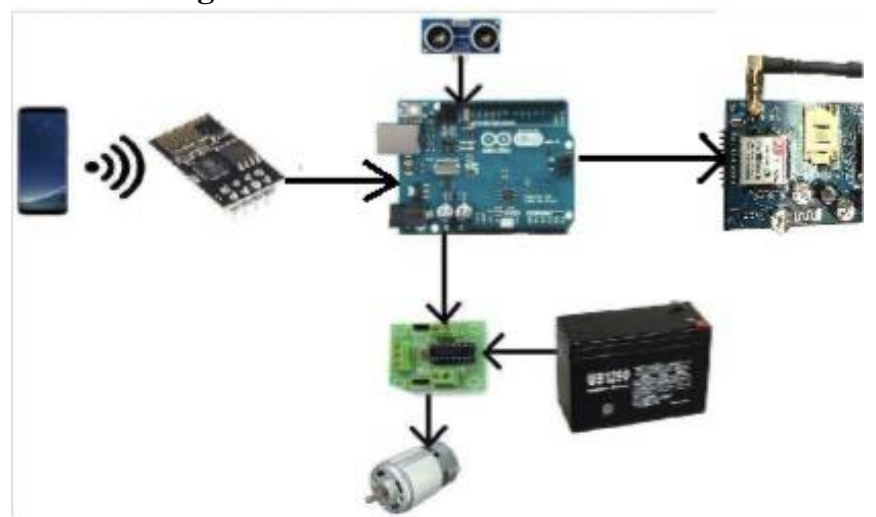

Fig. 7. Flow Diagram

Components used in the Fig 7 are as follows:

$>$ Smartphone

$>$ Wi-Fi module (ESP8266) (operating temperature

> Arduino UNO (i/p 7-12V, 6 analog pins, 14 digital pins)

$>$ Ultrasonic sensor (i/p $5 \mathrm{~V}$, operating current $<15 \mathrm{~mA}$, operating frequency $50 \mathrm{~Hz}$ )

> GSM Module 900A (dual band 900/1800 MHz, operating temperature $-40^{\circ} \mathrm{C}$ to $+85^{\circ} \mathrm{C}$ )

> Motor Driver (L293D) (supply voltage 4.5-36V, pulsed current 1.2-A)

$>$ Battery $(12 \mathrm{~V})$

$>$ DC motor

B. Project Setup

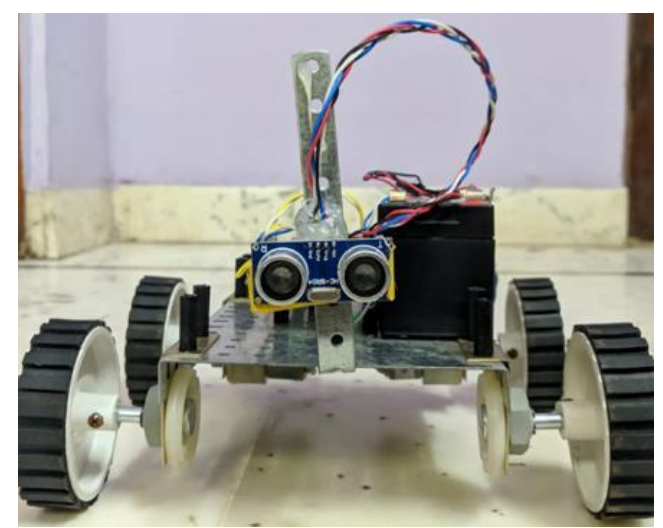

Fig. 8. Complete Project Setup - Front View 


\section{International Journal of Engineering Applied Sciences and Technology, 2019 Vol. 4, Issue 8, ISSN No. 2455-2143, Pages 350-355 \\ Published Online December 2019 in IJEAST (http://www.ijeast.com)}

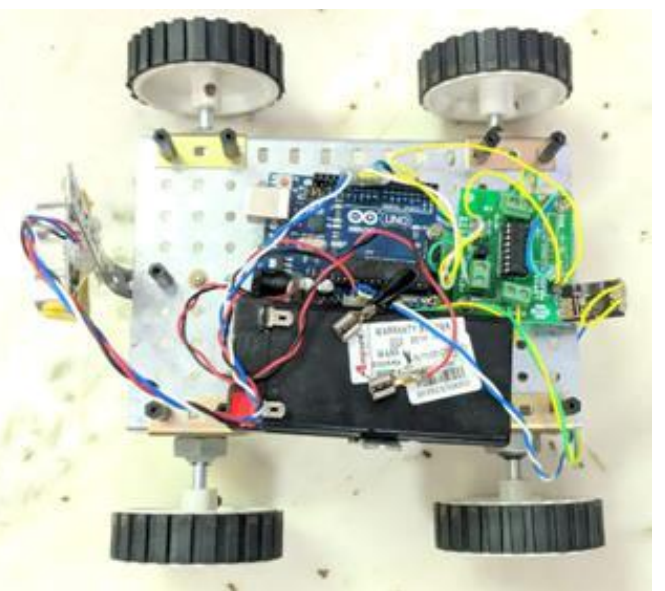

Fig. 9. Top View

\section{Procedure}

Smartphone contains Gyroscope, Magnetometer and Accelerometer sensors embedded into it for various applications. The software application, HyperIMU, accesses these sensor values and transfers it to the robot. The app detects linear acceleration using one or more accelerometers and rotational rate using one or more gyroscopes. Sometimes a magnetometer can also be included which is acts as a heading reference.

Typical configurations contain one accelerometer, gyroscope and magnetometer per axis for controlling the movement of the robot in left, right and forward directions. The same is supported by Barshan B. et al.(1995) in their work of Inertial Navigation Systems for mobile robots. From the work of Borenstein J., el al.(1988) obstacle and human detection is taken care by the Ultrasonic sensors mounted on the body of the robot to stop it from crashing. These sensors transmit ultrasonic signals and estimate the distance by determining the time required for reception of these signals after reflection from the target.

All these data are utilized to obtain the position of the human carrying the Smartphone. These data (from the Smartphone and Ultrasonic sensors) are given to the Arduino continuously in real-time. The Arduino collects the data processes it and then plans an executable instruction. This set of instructions then actuates the robot's motor drives thereby obtaining the following feature. Fig 8 and Fig 9 shows the completed project setup in front and top view.

Since the robot obediently follows the data as sent by the sensors of the Smartphone, it is possible to program it to detect any drastic variations in the sensory values thus obtained by it. This feature extends its potential to deal with healthcare-oriented applications of which one is Fall Detection. When the person carrying the Smartphone experiences an accidental fall there would be a large variation in the data, a spike, which would have a short period of duration of few seconds. Initializing thresholds while testing the Smartphone sensors for this wide variation of data among its coordinate axes helps us to determine an accidental fall. A short time delay is given to ensure that the person is able to recover from the fall. If otherwise, the robot would send an alert to the emergency contacts which would be pre-installed, indicating that the person has lost his conscious after a fall occurrence.

\section{RESULTS}

The Fig 11, Fig 12, Fig 13 shows real-time signal charts of Gyroscope, Magnetometer and Accelerometer sensors inbuilt into a Smartphone. The coordinate system of the Smartphone is defined by the screen orientation in which it is held by the person. The signal charts show variations along the axes when the mobile is rotated in the directions corresponding to the axes. According to this Android API as shown in Fig 10, X axis is horizontal, $\mathrm{Y}$ axis is vertical and $\mathrm{Z}$ axis points away from the front face of the phone.

The sensor data values are transmitted to the Arduino using standard internet protocols UDP or TCP via Wi-Fi. These data are sequential, and their order is same as that specified in the settings of the application. The stream of data is sent in a Comma Separated Value (CSV) format i.e., a single string consisting of all sensor values are separated by commas. At a time, three values corresponding to $\mathrm{X}, \mathrm{Y}$ and $\mathrm{Z}$ axes are sampled by the Smartphone application. For example, let three sensors be considered, the string of values passed through the stream will be -

“ $0.183,0.598,0.2468,0.205,-0.496,8.353,5.975,0.00,0.00$ $<\mathrm{CR}><\mathrm{LF}>$ "

$[\mathrm{CR}][\mathrm{LF}]$ inserted at the end of the stream indicate the termination of the stream.

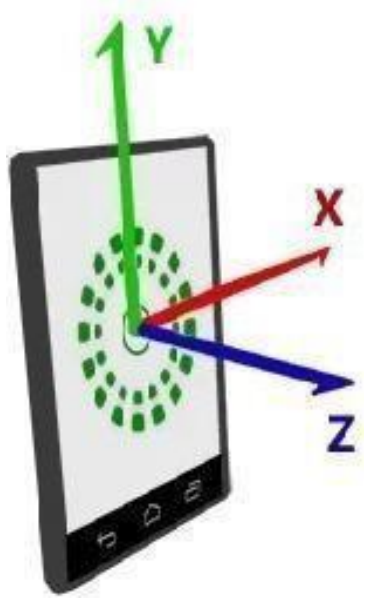

Fig. 10. Coordinate System of Smartphone 


\section{International Journal of Engineering Applied Sciences and Technology, 2019 Vol. 4, Issue 8, ISSN No. 2455-2143, Pages 350-355 \\ Published Online December 2019 in IJEAST (http://www.ijeast.com)}

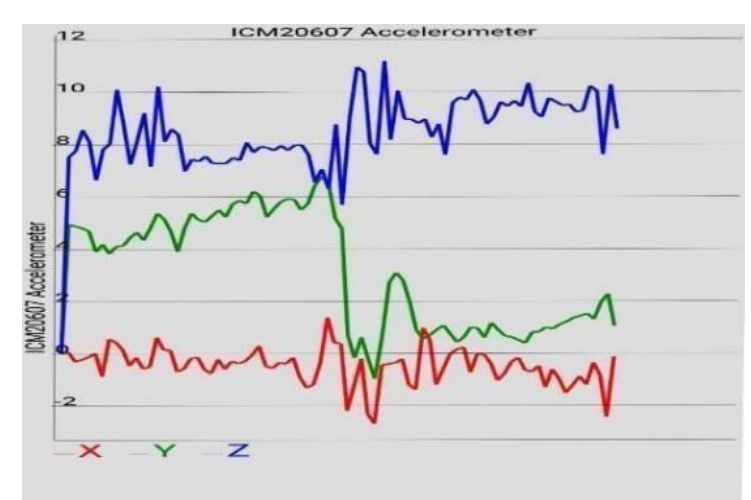

Fig. 11. Accelerometer Data

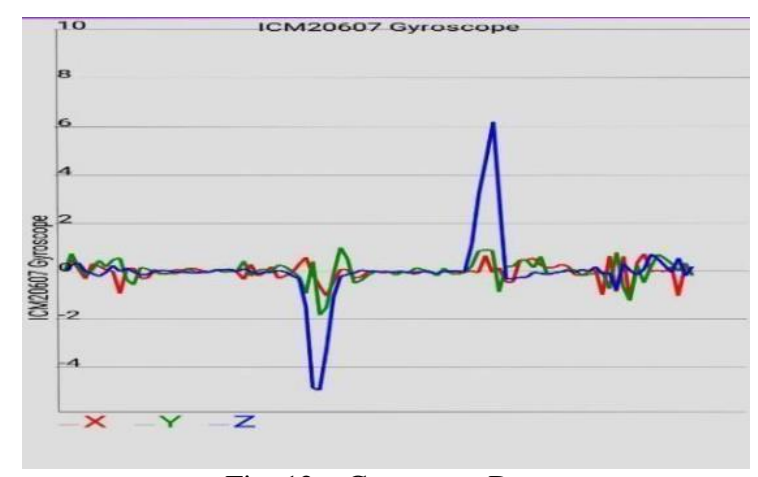

Fig. 12. Gyroscope Data

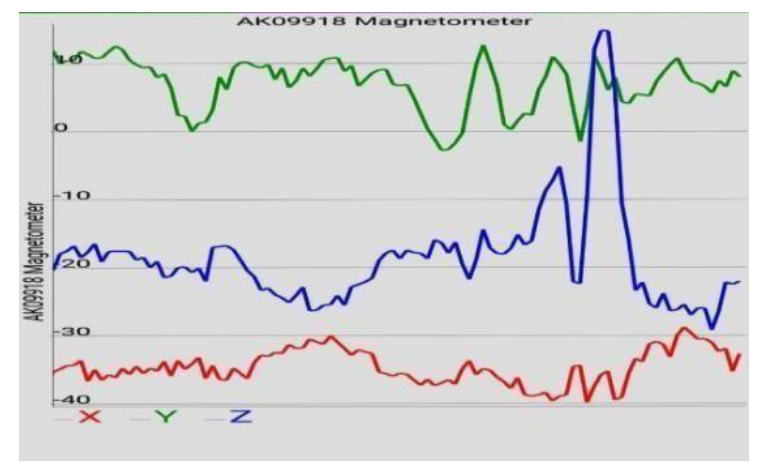

Fig. 13. Magnetometer Data

The entire string of data as received by the robot is filtered and parsed. It is then worked on within the Arduino IDE environment to obtain an appropriate plan of action. Based on these data values the robot estimates the position of the target human being followed. Ultimately it is programmed in such a way that the robot heads in that direction accordingly.

\section{Advantages}

$>$ The proposed project can be implemented not only for outdoor tracking but also indoors like malls, hospitals, offices, buildings, etc.

$>$ This human following robot is easily affordable and requires only minimal battery power to operate.
$>$ The robot can be implemented to follow aged people as timely and reliable surveillance is necessary to monitor their declining health issues, one of which is an accidental fall and the robot can alert about the same to the emergency contacts pre-installed.

$>$ The robot accesses the IMU data from the Smartphone which is ubiquitously available with everyone these days.

\section{CONCLUSION}

The study involving the use of robots in our personal lives assess the potential of robotic applications which are expected to create a tremendous value to professionals and households. The study offers insights from a point of cost effectiveness with the integration of robots within homes and other domains of human interaction that is nonindustrial in nature. With Artificial Intelligence and Machine Learning becoming more pervasive, robots of the future will be very intelligent with the ability to sense its environment, avoid obstacles, communicate, understand emotions, etc. This will allow robots to not only seamlessly move about in congested, crowded spaces e.g., malls, but they will also have the intelligence to make informed decisions in real time, thereby allowing them to fully function autonomously. This paper has challenged and opened up a plethora of robotic opportunities for home environments right from simple tasks such as cleaning to more complicated functions such as assisted elderly care, for children with special needs, etc.

\section{ACKNOWLEDGEMENT}

We would like to express our gratitude to our professor Dr. Mahesh K who motivated and constantly supported us. I also thank Sir MVIT for giving us an opportunity to work on a novel project which gave us a lot of research insights in this field.

\section{REFERENCE}

[1] Krueger V., Chazoule A., Crosby M., Lasnier A., Pedersen M. R., Rovida F., Veiga G. (2016). A Vertical and Cyber-Physical Integration of Cognitive Robots in Manufacturing. Proceedings of the IEEE, Vol. 104, No.5, 1114-1127.

[2] Pradeep B. V., Rahul E. S., \& Bhavani R. R. (2017). Follow me robot using bluetooth-based position estimation. 2017 International Conference on Advances in Computing, Communications and Informatics (ICACCI), doi:10.1109/icacci.2017.8125903

[3] Marina Md. Din., Jamil N., Maniam J., Mohamed A. M. (2018). Indoor positioning: technology comparison analysis. International Journal of Engineering \& Technology, Vol. 7, No. 2.14, 133-137. 


\section{International Journal of Engineering Applied Sciences and Technology, 2019 \\ Vol. 4, Issue 8, ISSN No. 2455-2143, Pages 350-355 \\ Published Online December 2019 in IJEAST (http://www.ijeast.com)}

[4] Sureshkumar R., Pathamuthu R. (2018). Android Mobile Phone Controlled Robot Using Internet of Things. International Journal of Pure and Applied Mathematics, Vol. 118, No. 5, 1031-1035.

[5] Honig S. S., Oron-Gilad T., Zaichyk H., SarneFleischmann V., Olatunji S., \& Edan Y. (2018). Towards Socially Aware Person-Following Robots. IEEE Transactions on Cognitive and Developmental Systems, 1-1.

[6] Ren L., \& Peng Y. (2019). Research of Fall Detection and Fall Prevention Technologies: A Systematic Review. IEEE Access, 1-1.

[7] Hu J.-S., Wang J.-J., \& Ho D. M. (2014). Design of Sensing System and Anticipative Behavior for Human Following of Mobile Robots. IEEE Transactions on Industrial Electronics, 61(4), 1916-1927.

[8] Kianifar R., Joukov V., Lee A., Raina S., \& Kulić D. (2019). Inertial measurement unit-based pose estimation: Analyzing and reducing sensitivity to sensor placement and body measures. Journal of Rehabilitation and Assistive Technologies Engineering, Vol. 6, 1-12.

[9] Morioka K., Lee J.-H., \& Hashimoto H. (2004). Human-Following Mobile Robot in a Distributed Intelligent Sensor Network. IEEE Transactions on Industrial Electronics, 51(1), 229-237.

[10] Barshan B. \& Durrant-Whyte H. F. (1995). Inertial Navigation Systems for Mobile Robots. IEEE Transactions on Robots and Automation, Vol. 11, No. 3, 328-342.

[11] Borenstein J., \& Koren Y. (1988). Obstacle avoidance with ultrasonic sensors. IEEE Journal on Robotics and Automation, 4(2), 213-218.

[12] Decker, M., Fischer, M., \& Ott, I. (2017). Service Robotics and Human Labor: A first technology assessment of substitution and cooperation. Robotics and Autonomous Systems, 87, 348-354.

[13] Fukuda T., Di P., Chen F., Sekiyama K., Huang J., Nakajima H. \& Kojima M. (2011). Advanced Service Robotics for Human Assistance and Support. International Conference of Advanced Computer Science Information System (ICACSIS), 2011 pp. 25-30. 